PROCEEDINGS OF THE

AMERICAN MATHEMATICAL SOCIETY

Volume 137, Number 1, January 2009, Pages 145-154

S 0002-9939(08)09660-3

Article electronically published on August 13, 2008

\title{
WHICH MEASURES ARE PROJECTIONS OF PURELY UNRECTIFIABLE ONE-DIMENSIONAL HAUSDORFF MEASURES
}

\author{
MARIANNA CSÖRNYEI AND VILLE SUOMALA \\ (Communicated by Tatiana Toro)
}

\begin{abstract}
We give a necessary and sufficient condition for a measure $\mu$ on the real line to be an orthogonal projection of $\mathcal{H}_{A}^{1}$ for some purely 1-unrectifiable planar set $A$.
\end{abstract}

\section{INTRODUCTION}

Let $A \subset \mathbb{R}^{2}$ be a purely 1-unrectifiable Borel set with $0<\mathcal{H}^{1}(A)<\infty$. The well-known projection results of Besicovitch and Marstrand (see, e.g., $\mathrm{Fa}$ or $\mathrm{Ma}$ ) tell us that for almost all $t \in \mathbb{R}$ the orthogonal projection of $\left.\mathcal{H}^{1}\right|_{A}$ to the line $\ell_{t}=\{(x, t x): x \in \mathbb{R}\}$ is singular with respect to the Lebesgue measure on $\ell_{t}$ and moreover has dimension 1 . These results, however, do not tell anything about one particular projection. In this paper we answer the following question of D. Preiss: for which measures $\mu$ on the real line is there a purely 1-unrectifiable Borel set $A \subset \mathbb{R}^{2}$ such that $\mu=\left.\operatorname{proj} \mathcal{H}^{1}\right|_{A}$ ? Here $\left.\mathcal{H}^{1}\right|_{A}$ is the one dimensional Hausdorff measure restricted to the set $A$. By proj we always mean the orthogonal projection proj: $\mathbb{R}^{2} \rightarrow \mathbb{R}$ onto the $x$-axis, $\operatorname{proj}(x, y)=x$, and if $\nu$ is a measure on $\mathbb{R}^{2}$ we define the projected measure $\operatorname{proj} \nu$ by defining $\operatorname{proj} \nu(A)=\nu\left(\operatorname{proj}^{-1}(A)\right)$ for all Borel sets $A \subset \mathbb{R}$.

Since any purely 1 -unrectifiable planar set $A$ intersects all vertical lines in a set of zero $\mathcal{H}^{1}$ measure it follows that if $\mu=\left.\operatorname{proj} \mathcal{H}^{1}\right|_{A}$, then

$$
\mu\{x\}=0 \text { for all } x \in \mathbb{R} ;
$$

that is, $\mu$ has no point masses. As another necessary condition we have

$$
\Theta^{1}(\mu, x):=\lim _{r \downarrow 0} \mu[x-r, x+r] /(2 r) \geq 1 \text { for } \mu \text {-almost all } x \in \mathbb{R} .
$$

To check that (1.2) holds we may assume that $\mu$ is absolutely continuous since $\Theta^{1}(\mu, x)=\infty$ almost everywhere on the singular part. Then it is easy to see that for $\mu$-almost all points $x \in \operatorname{proj}(A)$ we have

$$
\begin{aligned}
& \Theta^{1}(\mu, x)=\limsup _{\delta \downarrow 0}\{\mu(I) / \operatorname{diam}(I): x \in I \text { and } 0<\operatorname{diam}(I)<\delta\} \\
& \geq \limsup _{\delta \downarrow 0}\left\{\mathcal{H}^{1}(A \cap E) / \operatorname{diam}(E): y \in E, 0<\operatorname{diam}(E)<\delta\right\}
\end{aligned}
$$

Received by the editors November 23, 2007.

2000 Mathematics Subject Classification. Primary 28A78, 28A80.

(C)2008 American Mathematical Society Reverts to public domain 28 years from publication 
for all points $y \in A \cap \operatorname{proj}^{-1}\{x\}$. But the last quantity in (1.3), the upper convex density of $A$, is known to be at least one at $\mathcal{H}^{1}$-almost all $y \in A$; see [Fa, Theorem 2.3] for instance. This proves the necessity of (1.2).

Our main result, Theorem 1.1 below, shows that the necessary conditions (1.1) and (1.2) for $\mu$ are also sufficient.

Theorem 1.1. Suppose that $\mu$ is a locally finite measure on the real line satisfying (1.1) and (1.2). Then there is a purely 1-unrectifiable Borel set $A \subset \mathbb{R}^{2}$ for which $\mu=\left.\operatorname{proj} \mathcal{H}^{1}\right|_{A}$.

To prove Theorem 1.1 we divide $\mu$ into its singular and absolutely continuous parts and handle these separately. The singular part will be considered in $\$ 2$ and the absolutely continuous case is dealt with in 93 . Though we are mainly interested in projections of $\left.\mathcal{H}^{1}\right|_{A}$ for purely unrectifiable sets $A$, our methods may also be used to construct other fractal-type measures $\nu$ on $\mathbb{R}^{2}$ for which $\operatorname{proj} \nu=\mu$ for a given measure $\mu$ defined on $\mathbb{R}$; see Remark 2.4.

We end this introduction with some notation. We follow the usual convention according to which a measure on $\mathbb{R}^{n}$ always means a non-negative Borel regular outer measure defined on all subsets of $\mathbb{R}^{n}$. By a singular measure we mean a measure defined on $\mathbb{R}$ that is singular with respect to the Lebesgue measure $\mathcal{L}$. If $\nu$ and $\mu$ are finite measures on some $\mathbb{R}^{n}$, we denote $\nu \leq \mu$ if $\nu(A) \leq \mu(A)$ for all sets $A \subset \mathbb{R}^{n}$. In this case we may also consider the measure $\mu-\nu$ given by $(\mu-\nu)(A)=\mu(A)-\nu(A)$ for Borel sets $A \subset \mathbb{R}^{n}$.

\section{The SINGUlar CASE}

We begin with some notation needed in this section. If $k \in \mathbb{N}$, we call the collection of closed squares

$$
\mathcal{Q}_{k}=\left\{Q_{i, j}=\left[\frac{j-1}{k}, \frac{j}{k}\right] \times\left[\frac{i-1}{k}, \frac{i}{k}\right] \subset \mathbb{R}^{2}: 1 \leq i, j \leq k\right\}
$$

a $k$-grid. A collection of grid squares $\mathcal{Q} \subset \mathcal{Q}_{k}$ is called porous if it does not contain two neighboring squares, that is, $Q \cap Q^{\prime}=\emptyset$ whenever $Q, Q^{\prime} \in \mathcal{Q}$ and $Q \neq Q^{\prime}$.

The basis for our constructions is the following combinatorial lemma that enables us to find relatively good approximations for the set $A$ using $k$-adic squares when $k$ is so large that for "most" intervals $I_{j}=\left[\frac{j-1}{k}, \frac{j}{k}\right], 1 \leq j \leq k$, we have $1 / k \ll$ $\mu\left(I_{j}\right) \ll 1$.

Lemma 2.1. Let $\mathcal{Q} \subset \mathcal{Q}_{k}$ be an arbitrary collection of grid squares containing at most one square from each row $1 \leq i \leq k$. Denote by $l_{j}$ the number of squares that $\mathcal{Q}$ contains from the $j$ th column. If $l_{j}<k / 18$ for all $1 \leq j \leq k$, then there is a porous collection $\mathcal{Q}^{\prime} \subset \mathcal{Q}_{k}$ of grid squares that contains at most one square from each row and exactly $l_{j}$ squares from column $j$ for all $1 \leq j \leq k$.

Proof. If $Q \in \mathcal{Q}_{k}$, we denote by $N(Q)$ the union of $Q$ and its neighboring squares. Assume that $Q_{i_{0}, j_{0}} \in \mathcal{Q}$ has a neighboring square in the collection $\mathcal{Q}$. We define three index sets:

$$
\begin{aligned}
I & =\left\{1 \leq i \leq k: Q \subset N\left(Q_{i, j_{0}}\right) \text { for some } Q \in \mathcal{Q}\right\} \\
J & =\left\{1 \leq j \leq k: Q \subset N\left(Q_{i_{0}, j}\right) \text { for some } Q \in \mathcal{Q}\right\} \\
I^{\prime} & =\left\{1 \leq i \leq k: Q_{i, j} \in \mathcal{Q} \text { for some } j \in J\right\}
\end{aligned}
$$


Then $\# I \leq 3\left(l_{j_{0}-1}+l_{j_{0}}+l_{j_{0}+1}\right)<k / 2$ and also $\# J \leq 9$ since $\mathcal{Q}$ contains at most one square from each row $i_{0}-1, i_{0}$ and $i_{0}+1$. Moreover $\# I^{\prime}<\frac{k}{18} \# J \leq k / 2$.

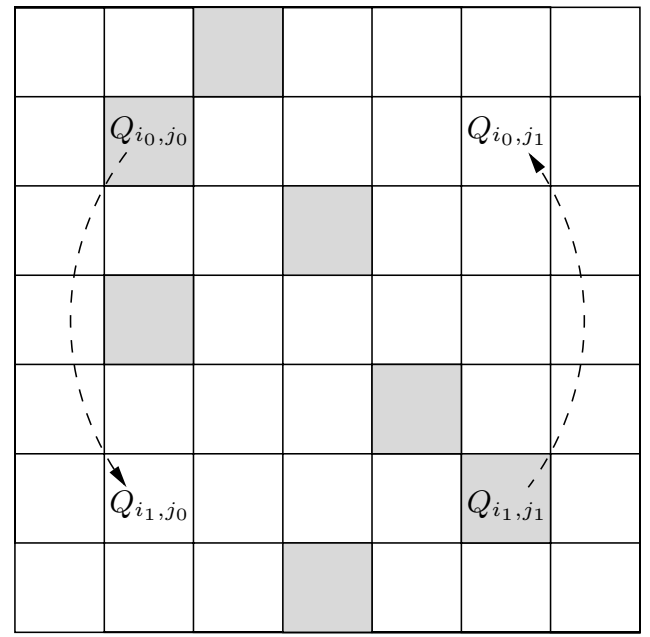

FiguRE 1. Constructing $\mathcal{Q}^{\prime}$ from $\mathcal{Q}$. The neighbors of $Q_{i_{1}, j_{0}}$ and $Q_{i_{0}, j_{1}}$ are not contained in the original collection $\mathcal{Q}$.

We now choose an index $i_{1} \in\{1, \ldots, k\} \backslash\left(I \cup I^{\prime}\right)$ and replace the square $Q_{i_{0}, j_{0}}$ in $\mathcal{Q}$ by $Q_{i_{1}, j_{0}}$. If $Q_{i_{1}, j_{1}} \in \mathcal{Q}$ for some $j_{1}$, we also replace the square $Q_{i_{1}, j_{1}}$ in $\mathcal{Q}$ by $Q_{i_{0}, j_{1}}$; see Figure 1. These replacements do not affect the good properties of $\mathcal{Q}$; it still contains at most one square from each row and exactly $l_{j}$ squares from the $j$ th column. But if the original collection had $m$ squares with some neighbors in $\mathcal{Q}$, the modified collection has at most $m-1$ squares with neighbors in $\mathcal{Q}$.

We repeat the above process inductively. It is clear that after a finite number of steps, we are left with a porous collection $\mathcal{Q}^{\prime}$ satisfying the requirements of the lemma.

The following lemma is merely a restatement of the singularity of $\mu$. We give the details for convenience.

Lemma 2.2. Suppose that $\mu$ is a singular measure on $[0,1]$ with no point masses. For $k \in \mathbb{N}$ and $1 \leq j \leq k$, we denote $m_{j}=\mu\left[\frac{j-1}{k}, \frac{j}{k}\right]$ and let $l_{j}$ be the greatest integer for which $l_{j} \leq k m_{j}$. Then $\lim _{k \rightarrow \infty} \sum_{j=1}^{k} l_{j} / k=\mu[0,1]$.

Proof. Let $\varepsilon>0$ and $M=5 \mu[0,1] / \varepsilon$. Since $\mu$ is singular, we have $\Theta^{1}(\mu, x)=$ $\lim _{r \downarrow 0} \mu[x-r, x+r] /(2 r)=\infty$ for $\mu$-almost all $x \in[0,1]$ and choosing $k_{0} \in \mathbb{N}$ large enough, we have $\mu\left(A_{k}\right)<\varepsilon$ for all $k \geq k_{0}$, where

$$
A_{k}=\left\{x \in[0,1]: \mu[x-1 / k, x+1 / k]<M^{2} / k\right\} .
$$

We now fix $k \geq k_{0}$ and choose a collection $J_{i}=\left(x_{i}-1 / k, x_{i}+1 / k\right), i=1, \ldots, N$, of non-overlapping intervals such that $x_{i} \in[0,1] \backslash A_{k}$ for all $i$ and $[0,1] \backslash A_{k} \subset$ $\bigcup_{i=1}^{N} 2 J_{i}=\bigcup_{i=1}^{N}\left(x_{i}-2 / k, x_{i}+2 / k\right)$. Letting $I_{j}=\left[\frac{j-1}{k}, \frac{j}{k}\right]$ for $1 \leq j \leq k$, we define

$$
B_{k}=\bigcup_{\mu\left(I_{j}\right)<M / k} I_{j}
$$


Since any of the intervals $2 J_{i}$ can intersect at most 5 of the intervals $I_{j}$ we have $\mu\left(B_{k} \cap 2 J_{i}\right) \leq 5 M / k \leq 5 \mu\left(J_{i}\right) / M$ for all $i$ and consequently

$$
\begin{aligned}
\mu\left(B_{k}\right) & =\mu\left(A_{k}\right)+\mu\left(B_{k} \backslash A_{k}\right) \leq \varepsilon+\sum_{i} \mu\left(B_{k} \cap 2 J_{i}\right) \\
& \leq \varepsilon+\frac{5}{M} \sum_{i} \mu\left(J_{i}\right) \leq \varepsilon+5 \mu[0,1] / M=2 \varepsilon .
\end{aligned}
$$

If $m_{j}=\mu\left(I_{j}\right) \geq M / k$ we have $l_{j} / k \geq(1-1 / M) \mu\left(I_{j}\right)$. Thus

$$
\begin{aligned}
\sum_{j=1}^{k} l_{j} / k & \geq \sum_{\mu\left(I_{j}\right) \geq M / k} l_{j} / k \geq \sum_{\mu\left(I_{j}\right) \geq M / k}(1-1 / M) \mu\left(I_{j}\right)=(1-1 / M) \mu\left([0,1] \backslash B_{k}\right) \\
& \geq(1-1 / M) \mu[0,1]-\mu\left(B_{k}\right) \geq \mu[0,1]-3 \varepsilon
\end{aligned}
$$

for all $k \geq k_{0}$. Letting $\varepsilon \downarrow 0$ we have the claim.

Our next step towards proving Theorem 1.1 is the following lemma.

Lemma 2.3. Let $\mu$ be a finite and singular measure on $[0,1]$ with no point masses and let $\delta>0$. Then there is a purely 1 -unrectifiable Borel set $A \subset[0,1] \times[0, \delta]$ such that $\mathcal{H}^{1}(A) \geq \mu[0,1] / 2$ and $\left.\operatorname{proj} \mathcal{H}^{1}\right|_{A} \leq \sqrt{2} \mu$.

Proof. We first note that we may assume without loss of generality that $\mu[0,1] \leq$ $\delta=1$. Indeed, in the general case, we may first choose $k \in \mathbb{N}$ so large that $k>1 / \delta$ and $m_{j}=\mu\left[\frac{j-1}{k}, \frac{j}{k}\right]<\delta / 2$ for all $1 \leq j \leq k$ and denote by $l_{j}$ the greatest integer for which $l_{j} \leq m_{j} k$. Then we can write $\left.\mu\right|_{\left[\frac{j-1}{k}, \frac{j}{k}\right]}=\sum_{i=1}^{l_{j}+1} \mu_{i, j}$ so that $\mu_{i, j}\left[\frac{j-1}{k}, \frac{j}{k}\right] \leq$ $1 / k$ for each $i, j$, and, using a rescaled version of the statement, we can find purely unrectifiable sets $A_{i, j} \subset\left[\frac{j-1}{k}, \frac{j}{k}\right] \times\left[\frac{i-1}{k}, \frac{i}{k}\right]$ so that $\mathcal{H}^{1}\left(A_{i, j}\right) \geq \mu_{i, j}\left[\frac{j-1}{k}, \frac{j}{k}\right] / 2$ and $\left.\operatorname{proj} \mathcal{H}^{1}\right|_{A_{i, j}} \leq \sqrt{2} \mu_{i, j}$. Since the squares $\left[\frac{j-1}{k}, \frac{j}{k}\right] \times\left[\frac{i-1}{k}, \frac{i}{k}\right]$ are non-overlapping and they are inside $[0,1] \times\left[0, \frac{l_{j}+1}{k}\right]$, where $\frac{l_{j}+1}{k} \leq \max \left(1,2 l_{j}\right) / k<\delta$, we can take $A=\bigcup_{i, j} A_{i, j} \subset[0,1] \times[0, \delta]$.

Let $\mu[0,1] \leq \delta=1$. We construct a set $A \subset[0,1] \times[0,1]$ by iterative use of Lemma 2.1] First choose numbers $\varepsilon_{s}>0$ for $s \in \mathbb{N}$ such that $\sum_{s=1}^{\infty} \varepsilon_{s} \leq \mu[0,1] / 2$.

Step 1: Given $k \in \mathbb{N}$, define $m_{j}=m_{j, k}=\mu\left[\frac{j-1}{k}, \frac{j}{k}\right]$ for all $1 \leq j \leq k$ and let $l_{j}$ be the greatest integer satisfying $l_{j} \leq m_{j} k$. By Lemma 2.2 we may choose $k=k_{1}$ large enough so that $\sum_{j=1}^{k} l_{j} / k>\mu[0,1]-\varepsilon_{1}$. Increasing $k$ if necessary, we may also assume that $l_{j}<k / 18$ for all $1 \leq j \leq k$ since $\mu$ contains no point masses. Let $\mathcal{Q}=\left\{Q_{i, j}: 1 \leq j \leq k_{1}, \sum_{r=1}^{j-1} l_{r}<i \leq \sum_{r=1}^{j} l_{r}\right\} \subset \mathcal{Q}_{k_{1}}$. Then $\mathcal{Q}$ satisfies the assumptions of Lemma 2.1. Thus we may find a porous collection of $k_{1}$-grid squares $\mathcal{R}^{1} \subset \mathcal{Q}_{k_{1}}$ that contains exactly $l_{j}$ squares from the $j$ th column and at most one square from each row. Let $A_{1}=\bigcup_{Q \in \mathcal{R}^{1}} Q$ be the union of all these squares.

Step $n$ : Suppose that we are given a collection $\mathcal{R}^{n-1} \subset \mathcal{Q}_{k}$ of porous $k$-grid squares, $k=k_{n-1}$, that contains at most one square from each row and $l_{j}=l_{j, n-1}$ squares from the $j$ th column such that $l_{j, n-1} / k_{n-1} \leq \mu\left[\frac{j-1}{k_{n-1}}, \frac{j}{k_{n-1}}\right]$ and

$$
\sum_{j=1}^{k_{n-1}} l_{j, n-1} / k_{n-1} \geq \mu[0,1]-\sum_{s=1}^{n-1} \varepsilon_{s} .
$$


Consider one of the squares $Q=Q_{i^{\prime}, j^{\prime}} \in \mathcal{R}^{n-1} \subset \mathcal{Q}_{k_{n-1}}$ and define $\widetilde{\mu}=$ $\left.\left(k_{n-1} \mu\left[\frac{j^{\prime}-1}{k_{n-1}}, \frac{j^{\prime}}{k_{n-1}}\right]\right)^{-1} \mu\right|_{\left[\frac{j^{\prime}-1}{k_{n-1}}, \frac{j^{\prime}}{k_{n-1}}\right]}$. We now perform the Step 1 construction inside $Q$ replacing $[0,1] \times[0,1]$ by $Q$ and $\mu$ by $\widetilde{\mu}$. Observe that the total mass of $\widetilde{\mu}$ is $1 / k_{n-1}$. Defining $m_{j}=\widetilde{\mu}\left[\frac{j^{\prime}-1}{k_{n-1}}+\frac{j-1}{k^{\prime} k_{n-1}}, \frac{j^{\prime}-1}{k_{n-1}}+\frac{j}{k^{\prime} k_{n-1}}\right]$ and $l_{j}^{\prime}$ as the largest integer for which $l_{j}^{\prime} \leq k^{\prime} k_{n-1} m_{j}$ it follows as in Step 1 that $l_{j}^{\prime}<k^{\prime} / 18$ for all $1 \leq j \leq k^{\prime}$ and

$$
\sum_{j=1}^{k^{\prime}} l_{j}^{\prime} /\left(k^{\prime} k_{n-1}\right)>\widetilde{\mu}(Q)-\varepsilon_{n} / k_{n-1}=\left(1-\varepsilon_{n}\right) / k_{n-1}
$$

provided $k^{\prime} \in \mathbb{N}$ is chosen large enough. Here the numbers $l_{j}^{\prime}$ actually depend on $j, j^{\prime}, n$ and also on $k^{\prime}$ but since there are only finitely many columns in $\mathcal{R}^{n-1}$, we may choose the same $k^{\prime} \in \mathbb{N}$ for all $Q \in \mathcal{R}^{n-1}$. Using Lemma 2.1 as above, we find a porous collection $\mathcal{Q}^{\prime}=\mathcal{Q}_{i^{\prime}, j^{\prime}}^{\prime} \subset \mathcal{Q}_{k^{\prime} k_{n-1}}$ of subsquares of $Q_{i^{\prime}, j^{\prime}}$ containing at most one square from each row and exactly $l_{j}^{\prime}$ squares from the $j$ th column of $Q_{i^{\prime}, j^{\prime}}$ (in the grid $\mathcal{Q}_{k^{\prime} k_{n-1}}$ ) for each $1 \leq j \leq k^{\prime}$. We finally define $k_{n}=k^{\prime} k_{n-1}$, let $\mathcal{R}^{n}=\bigcup_{Q \in \mathcal{R}^{n-1}} \mathcal{Q}^{\prime}$ denote the union of all the squares chosen inside the squares of $\mathcal{R}^{n-1}$, and define $A_{n}=\bigcup_{Q \in \mathcal{R}^{n}} Q$. It is easy to check that $\mathcal{R}^{n}$ has the same good properties as $\mathcal{R}^{n-1}$. Namely, it is porous, contains at most one square from each row and $l_{j}=l_{j, n}$ squares from the $j$ th column such that $l_{j, n} / k_{n} \leq \mu\left[\frac{j-1}{k_{n}}, \frac{j}{k_{n}}\right]$ for all $1 \leq j \leq k_{n}$. Moreover,

$$
\begin{gathered}
\sum_{j=1}^{k_{n}} l_{j, n} / k_{n}=\sum_{Q \in \mathcal{R}^{n-1}} \sum_{j=1}^{k^{\prime}} l_{j}^{\prime} /\left(k^{\prime} k_{n-1}\right)>\# \mathcal{R}^{n-1}\left(1-\varepsilon_{n}\right) / k_{n-1} \\
=\left(1-\varepsilon_{n}\right) \sum_{j=1}^{k_{n-1}} l_{j, n-1} / k_{n-1} \geq \mu[0,1]-\sum_{s=1}^{n} \varepsilon_{s},
\end{gathered}
$$

using (2.2) and (2.1). Here $\# \mathcal{R}^{n-1}$ denotes the number of elements in the collection $\mathcal{R}^{n-1}$. Observe the different roles of the numbers $l_{j}$ and $l_{j}^{\prime}$ : Above $l_{j}=l_{j, n}$ gives the total number of squares in the $j$ th column of the whole collection $\mathcal{R}^{n} \subset \mathcal{Q}_{k_{n}}$ whereas $l_{j}^{\prime}=l_{j, j^{\prime}}^{\prime}$ refers to the number of subsquares selected in the $j$ th column of a fixed subsquare $Q_{i^{\prime}, j^{\prime}} \in \mathcal{R}^{n-1}$. They are, however, related by the identity $l_{\left(j^{\prime}-1\right) k^{\prime}+j, n}=l_{j^{\prime}, n-1} l_{j}^{\prime}$ for $1 \leq j^{\prime} \leq k_{n-1}$ and $1 \leq j \leq k^{\prime}$.

Having defined all the sets $A_{n}$ inductively, we eventually let $A=\bigcap_{n} A_{n}$. It remains to show that $A$ is purely 1-unrectifiable and that it has the desired properties $\mathcal{H}^{1}(A) \geq \mu[0,1] / 2$ and proj $\left.\mathcal{H}^{1}\right|_{A} \leq \sqrt{2} \mu$. We start from the pure unrectifiability of $A$. Suppose that $\Gamma \subset \mathbb{R}^{2}$ is a $C^{1}$-curve. Since the collections $\mathcal{R}^{n}$ are porous for all $n \in \mathbb{N}$, it follows that the set $\Gamma \cap A$ has no density points, i.e. points $x \in \Gamma \cap A$ for which $\lim _{r \downarrow 0} \mathcal{H}^{1}\{y \in \Gamma \cap A:|x-y|<r\} /(2 r)=1$. This implies that $\mathcal{H}^{1}(\Gamma \cap A)=0$ and thus $A$ is purely 1-unrectifiable.

Recall that $\mathcal{R}^{n}$ contains at most one square from each row; hence $A$ contains at most one point on each, except possibly for countably many, horizontal lines. Let $\operatorname{proj}_{2}$ denote the projection to the $y$-axis $(x, y) \rightarrow y$, and let $\nu$ be the measure defined by $\nu(B)=\mathcal{H}^{1}\left(\operatorname{proj}_{2}(A \cap B)\right)$. Since projection cannot increase the $\mathcal{H}^{1}$ measure, it is clear that $\nu \leq\left.\mathcal{H}^{1}\right|_{A}$. It is also easy to see that $\nu \geq\left.\frac{1}{\sqrt{2}} \mathcal{H}^{1}\right|_{A}$ : indeed, $\mathcal{R}^{n}$ contains at most one square from each row; hence for $k=k_{n}$ and for each 
interval $I=\left(\frac{i-1}{k}, \frac{i}{k}\right), A \cap\left(\operatorname{proj}_{2}^{-1} I\right)$ can be covered by a square of side length $1 / k$, i.e. of diameter $\sqrt{2} / k$. Therefore it is enough to show that $\nu(A) \geq \mu[0,1] / 2$ and $\operatorname{proj} \nu \leq \mu$.

The first inequality follows immediately from

$$
\nu(A)=\mathcal{H}^{1}\left(\operatorname{proj}_{2} A\right)=\lim _{n \rightarrow \infty} \mathcal{H}^{1}\left(\operatorname{proj}_{2} A_{n}\right)
$$

and

$$
\mathcal{H}^{1}\left(\operatorname{proj}_{2} A_{n}\right)=\sum_{j=1}^{k_{n}} l_{j, n} / k_{n} \geq \mu[0,1]-\sum_{s=1}^{n} \varepsilon_{s} \geq \mu[0,1] / 2 .
$$

The second inequality follows from the fact that for each $k=k_{n}$, above each interval $J=\left(\frac{j-1}{k}, \frac{j}{k}\right)$, the set $A$ is covered by $l_{j}$ squares of $\mathcal{R}^{n}$ of side length $1 / k$; hence $\nu\left(A \cap \operatorname{proj}^{-1}(J)\right) \leq l_{j} / k \leq m_{j}=\mu(J)$.

To prove Theorem 1.1 for singular $\mu$ we still have to show how to find a purely unrectifiable $A \subset \mathbb{R}^{2}$ such that the measures proj $\left.\mathcal{H}^{1}\right|_{A}$ and $\mu$ are the same. An immediate corollary of Lemma 2.3 is that for any singular measure $\mu$ on $[0,1]$ with no point masses and for any $\delta>0$ there is a purely unrectifiable $A \subset[0,1] \times[0, \delta]$ for which $\mathcal{H}^{1}(A) \geq 2^{-3 / 2} \mu[0,1]$ and $\left.\operatorname{proj} \mathcal{H}^{1}\right|_{A} \leq \mu$.

Proof of Theorem 1.1 when $\mu$ is singular. Without loss of generality we can assume that $\mu$ is supported on $[0,1]$. First we choose a purely unrectifiable set $A_{1} \subset[0,1] \times[0,1 / 2]$ so that $\mathcal{H}^{1}\left(A_{1}\right) \geq 2^{-3 / 2} \mu[0,1]$ and proj $\left.\mathcal{H}^{1}\right|_{A_{1}} \leq \mu$. Then consider $\mu_{2}=\mu-\left.\operatorname{proj} \mathcal{H}^{1}\right|_{A_{1}}$ and choose a purely unrectifiable $A_{2} \subset[0,1] \times[1 / 2,3 / 4]$ for which $\mathcal{H}^{1}\left(A_{2}\right) \geq 2^{-3 / 2} \mu_{2}[0,1]$ and $\left.\operatorname{proj} \mathcal{H}^{1}\right|_{A_{2}} \leq \mu_{2}$. Proceeding in this manner we get purely unrectifiable sets $A_{n} \subset[0,1] \times\left[\left(1-2^{-n+1}\right),\left(1-2^{-n}\right)\right]$ and corresponding measures $\mu_{n}$ so that $\mathcal{H}^{1}\left(A_{n}\right) \geq 2^{-3 / 2} \mu_{n}[0,1]$, proj $\left.\mathcal{H}^{1}\right|_{A_{n}} \leq \mu_{n}$ and $\mu_{n+1}=\mu_{n}-$ proj $\left.\mathcal{H}^{1}\right|_{A_{n}}$. Then clearly $\mu_{n+1}[0,1] \leq\left(1-2^{-3 / 2}\right) \mu_{n}[0,1]$ for each $n$, in particular, $\mu_{n}[0,1] \rightarrow 0$. Since $\mu=\left.\sum_{i=1}^{n} \operatorname{proj} \mathcal{H}^{1}\right|_{A_{i}}+\mu_{n+1}$, this shows $\mu=\left.\sum_{i=1}^{\infty} \operatorname{proj} \mathcal{H}^{1}\right|_{A_{i}}$. Since the sets $A_{i}$ are purely unrectifiable and they are contained in pairwise nonoverlapping rectangles, for $A=\bigcup_{i=1}^{\infty} A_{i}, \mu=\left.\sum_{i=1}^{\infty} \operatorname{proj} \mathcal{H}^{1}\right|_{A_{i}}=\left.\operatorname{proj} \mathcal{H}^{1}\right|_{A}$.

Remark 2.4. The method presented above may also be used to construct other fractal-type measures $\nu$ on $\mathbb{R}^{2}$ such that $\operatorname{proj} \nu=\mu$ for a given locally finite measure $\mu$. At least the following statements may be obtained:

(1) If $0<s<1$ and $\Theta^{s}(\mu, x)=\lim _{r \downarrow 0} \mu[x-r, x+r] /(2 r)^{s}=\infty$ for $\mu$-almost all $x \in \mathbb{R}$, then there is a Borel set $A \subset \mathbb{R}^{2}$ such that $\mu=\left.\operatorname{proj} \mathcal{H}^{s}\right|_{A}$.

(2) If $s>1$ and $\Theta^{*(s-1)}(\mu, x)=\limsup _{r \downarrow 0} \mu[x-r, x+r] /(2 r)^{s-1}<\infty$ for $\mu$ almost all $x \in \mathbb{R}$, then there is a Borel set $A \subset \mathbb{R}^{2}$ such that $\mu=\left.\operatorname{proj} \mathcal{H}^{s}\right|_{A}$.

To prove (11) one uses the following simple observation in place of Lemma 2.1 (the notation is as in Lemma 2.3): If $Q$ is a collection of $k$-grid squares such that $\sum_{j=1}^{k} l_{j} \leq k^{s}$, then there is a collection $\mathcal{Q}^{\prime}$ containing exactly $l_{j}$ squares from the $j$ th column such that $\#\left\{Q \in \mathcal{Q}^{\prime}: B \cap Q \neq \emptyset\right\} \leq C k^{s} \operatorname{diam}(B)^{s}$ for all balls $B \subset \mathbb{R}^{2}$ such that $\frac{1}{k} \leq \operatorname{diam}(B) \leq 1$. To prove (2) we observe that a similar statement holds true if $s>1$ and $\sum_{j=j_{0}}^{j_{1}} l_{j} \leq C k\left(j_{1}-j_{0}\right)^{s-1}$ for all $1 \leq j_{0} \leq j_{1} \leq k$. This is seen just by distributing the $l_{j}$ squares in the $j$ th column evenly along the rows $1 \leq i \leq k$. 


\section{The ABsolutely COntinuous CASE}

In this section we prove Theorem 1.1 for $\mu$ that is absolutely continuous with respect to the Lebesgue measure $\mathcal{L}$. Let us begin with some preparations. For $\lambda>0$ we define similitudes $f_{i}^{\lambda}: \mathbb{R}^{2} \rightarrow \mathbb{R}^{2}$ for $i=1,2,3$ by the formulas $f_{1}^{\lambda}(x, y)=$ $\frac{1}{3}(x, y)+(0,0), f_{2}^{\lambda}(x, y)=\frac{1}{3}(x, y)+\left(\frac{1}{3}, \lambda \frac{2}{3}\right)$, and $f_{3}^{\lambda}(x, y)=\frac{1}{3}(x, y)+\left(\frac{2}{3}, \lambda \frac{1}{3}\right)$. Let $C_{\lambda} \subset[0,1] \times[0, \lambda]$ be the self-similar set induced by the similitudes $f_{i}^{\lambda}$; see Figure 2 .

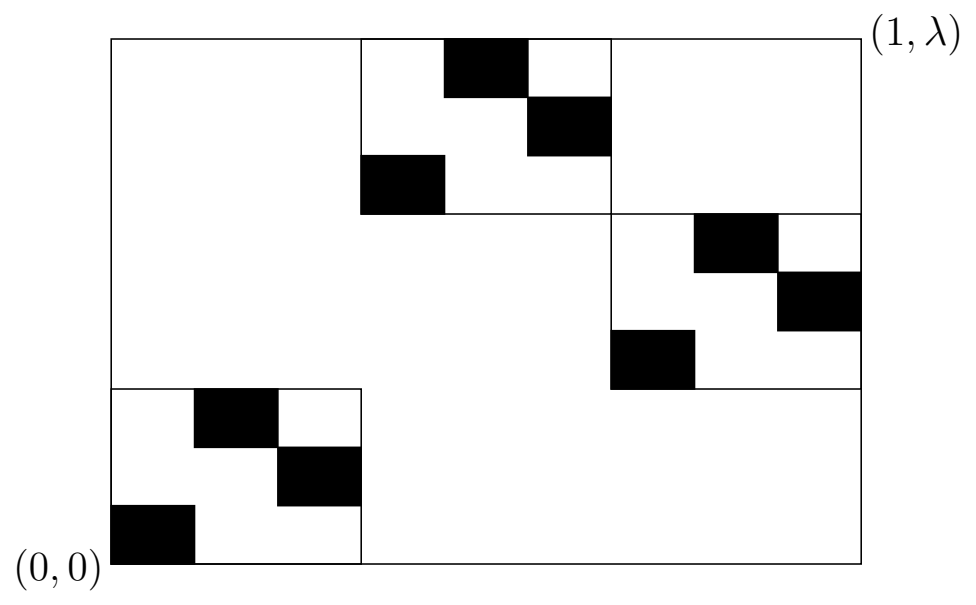

Figure 2. The set $C_{\lambda}$.

Define $h(\lambda)=\mathcal{H}^{1}\left(C_{\lambda}\right)$. Since the projection of $C_{\lambda}$ to the $y$-axis has length $\lambda$ we have $h(\lambda) \geq \lambda$; in particular, $\lim _{\lambda \rightarrow \infty} h(\lambda)=\infty$. It is also easy to see that $\lim _{\lambda \downarrow 0} h(\lambda)=1$. For all $0<\lambda_{0}, \lambda_{1}<\infty$ the set $C_{\lambda_{1}}$ is obtained from $C_{\lambda_{0}}$ by the vertical stretching/flattening $(x, y) \mapsto\left(x, \frac{\lambda_{1}}{\lambda_{0}} y\right)$ and we observe that $h$ is continuous and non-decreasing. It is also useful to note that if $\nu$ is the natural probability measure on $C_{\lambda}$, then $\operatorname{proj} \nu=\left.\mathcal{L}\right|_{[0,1]}$, and since $\left.\mathcal{H}^{1}\right|_{C_{\lambda}}=h(\lambda) \nu$ we see that proj $\left.\mathcal{H}^{1}\right|_{C_{\lambda}}=h(\lambda) \mathcal{L}$.

For any $0<\lambda<\infty$ we define an operation $\mathcal{O}_{\lambda}$ on all rectangles $R=(x, y)+$ $\left[0, l_{x}\right] \times\left[0, l_{y}\right] \subset \mathbb{R}^{2}$ for which $l_{y} \geq \lambda l_{x}$ by the formula

$$
\mathcal{O}_{\lambda}(R)=(x, y)+l_{x}\left(\bigcup_{i=1}^{3} f_{i}^{\lambda}([0,1] \times[0, \lambda])\right)
$$

Observe that then $\mathcal{O}_{\lambda}(R) \subset R$. We now define the increasing function $g:(1, \infty) \rightarrow$ $(0, \infty)$ by $g(t)=\max h^{-1}(\{t\})$ for all $t>1$. (If $h$ is one to one we can simply take $g=h^{-1}$ and then $g$ is continuous but we do not know if this is the case.)

Proof of Theorem 1.1 when $\mu$ is absolutely continuous. We assume that spt $\mu \subset$ $[0,1]$ and let $\Theta(x)=\Theta^{1}(\mu, x)$ denote the density of $\mu$ at $x$. Since $\mu$ is absolutely continuous, it follows that $\Theta(x)<\infty$ for almost every $x \in[0,1]$. For simplicity, we assume that $\Theta$ is continuous and that $\Theta^{-1}\{t\}$ has measure zero for all $t \geq 1$. The general case reduces to this as discussed at the end of the proof. 
The purely unrectifiable set $A$ is now constructed in the following manner. Let $t_{\max }=\max _{x \in[0,1]} \Theta(x)$ and $A_{0}=[0,1] \times\left[0, g\left(t_{\max }\right)\right]$. Suppose that $A_{k}=\bigcup_{j=1}^{3^{k}} R_{j}^{k}$ has been defined, where $R_{j}^{k}=\left[(j-1) 3^{-k}, j 3^{-k}\right] \times J_{j}^{k}$ for all $1 \leq j \leq 3^{k}$ and $3^{k} \ell\left(J_{j}^{k}\right) \geq g\left(t_{j}\right)$ and where $t_{j}=\max _{x \in\left[(j-1) 3^{-k}, j 3^{-k}\right]} \Theta(x)$. We then define

$$
A_{k+1}=\bigcup_{j=1}^{3^{k}} \mathcal{O}_{g\left(t_{j}\right)}\left(R_{j}^{k}\right)
$$

and finally $A=\bigcap_{k} A_{k}$. Then $A$ is purely 1-unrectifiable, which can be seen by looking at the set $A_{t}=A \cap \operatorname{proj}^{-1}\left(\Theta^{-1}(t, \infty)\right)$ for a fixed $t>1$ : The set $\Theta^{-1}(t, \infty) \subset$ $[0,1]$ is an open set and if $I \subset \Theta^{-1}(t, \infty)$ is a triadic interval of length $3^{-j}$, the set $A \cap \operatorname{proj}^{-1}(I)$ consists of three distinct parts so that the distance between any two of them is at least $\min \left\{\frac{1}{9}, \frac{g(t)}{3}\right\} 3^{-j}$. It follows as in the proof of Lemma 2.3 that no $C^{1}$-curve $\Gamma$ can intersect $A_{t}$ in a set of positive measure. Since $A_{t} \subset A$ for all $t>1$ and $\mathcal{H}^{1}\left(A_{t}\right) \rightarrow \mathcal{H}^{1}\left(A_{1}\right)$ as $t \rightarrow 1$ it follows that $A$ is purely 1-unrectifiable. Recall that we assumed that the level sets of $\Theta$, in particular $\Theta^{-1}\{1\}$, have measure zero.

To complete the proof we have to show that $\left.\operatorname{proj} \mathcal{H}^{1}\right|_{A}=\mu$. This will be done using the following lemma.

Lemma 3.1. Let $1<t<\infty, \varepsilon>0$, and $B_{t, \varepsilon}=\Theta^{-1}(t, t+\varepsilon)$. Then $\left.\frac{1}{c} \mu\right|_{B_{t, \varepsilon}} \leq$ $\left.\left(\left.\operatorname{proj} \mathcal{H}^{1}\right|_{A}\right)\right|_{B_{t, \varepsilon}} \leq\left. c \mu\right|_{B_{t, \varepsilon}}$, where

$$
c=1+54(g(t+\varepsilon)-g(t)) / \min \{1,3 g(t)\} .
$$

Proof. We begin with a technical remark. Let $E \subset[0,1]$ denote the countable set consisting of the endpoints of all triadic intervals $I \subset[0,1]$. Since $A$ is purely 1-unrectifiable, the measure $\mathcal{H}^{1}(A)$ does not change if we remove the vertical lines $\operatorname{proj}^{-1}\{x\}$ from the set $A$ for all $x \in E$. This makes the mapping $x \mapsto \operatorname{proj} x$, $A \rightarrow[0,1] \backslash E$ one to one. For a given $\lambda>0$ we do the same for the set $C_{\lambda}$, that is, remove the vertical lines $\operatorname{proj}^{-1}\{x\}$ from $C_{\lambda}$ for all $x \in E$. After this we can define a natural bijection between $A$ and $C_{\lambda}$ by demanding that $x \mapsto x^{\prime}$ if and only if $\operatorname{proj}\left(x^{\prime}\right)=\operatorname{proj}(x)$.

Since $B_{t, \varepsilon}$ is an open set it is enough to show that $\frac{1}{c} \mu(I) \leq\left(\left.\operatorname{proj} \mathcal{H}^{1}\right|_{A}\right)(I) \leq$ $c \mu(I)$ for any triadic interval $I \subset B_{t, \varepsilon}$ and by scaling this reduces to showing that $\frac{1}{c} \mu[0,1] \leq \mathcal{H}^{1}(A) \leq c \mu[0,1]$ assuming $B_{t, \varepsilon}=[0,1]$.

Let $x, y \in A, x \neq y$ and $x_{j}, y_{j} \in\{0,1,2\}$ be such that $\operatorname{proj} x=\sum_{j=1}^{\infty} x_{j} 3^{-j}$ and $\operatorname{proj} y=\sum_{j=1}^{\infty} y_{j} 3^{-j}$. We define $\lambda_{x}^{j}=g\left(\max _{x \in I_{x}^{j}} \Theta(x)\right)$, where $I_{x}^{j}$ is the unique triadic interval of size $3^{-j}$ containing $x$. The numbers $\lambda_{y}^{j}$ are defined in a similar manner. Now $\operatorname{proj}_{2} x=\sum_{j=1}^{\infty} \lambda_{x}^{j-1} x_{j}^{\prime} 3^{-j}$, where the mapping $x_{j} \mapsto x_{j}^{\prime}$ is defined by the rules $0 \mapsto 0,1 \mapsto 2,2 \mapsto 1$. Similarly $\operatorname{proj}_{2} y=\sum_{j=1}^{\infty} \lambda_{y}^{j-1} y_{j}^{\prime} 3^{-j}$. Recall that $\operatorname{proj}_{2}$ denotes the orthogonal projection onto the vertical coordinate axis.

Let $j_{0}$ be the smallest integer for which $I_{x}^{j_{0}} \neq I_{y}^{j_{0}}$ and let $x^{\prime}, y^{\prime} \in C_{g(t)}$ so that $\operatorname{proj} x=\operatorname{proj} x^{\prime}$ and $\operatorname{proj} y=\operatorname{proj} y^{\prime}$. Then

$$
\left|x^{\prime}-y^{\prime}\right| \geq \min \left\{\frac{1}{9}, \frac{g(t)}{3}\right\} 3^{-j_{0}}
$$


since $\operatorname{dist}\left(f_{i}^{g(t)}\left(C_{g(t)}\right), f_{j}^{g(t)}\left(C_{g(t)}\right)\right) \geq \min \left\{\frac{1}{9}, \frac{g(t)}{3}\right\}$ whenever $i, j \in\{1,2,3\}$ and $i \neq j$. Moreover

$$
\begin{aligned}
& \left|(x-y)-\left(x^{\prime}-y^{\prime}\right)\right|=\left|\operatorname{proj}_{2}\left(x-x^{\prime}\right)-\operatorname{proj}_{2}\left(y-y^{\prime}\right)\right| \\
& =\left|\left(\sum_{j=j_{0}}^{\infty}\left(\lambda_{x}^{j-1}-g(t)\right) x_{j}^{\prime} 3^{-j}\right)-\left(\sum_{j=j_{0}}^{\infty}\left(\lambda_{y}^{j-1}-g(t)\right) y_{j}^{\prime} 3^{-j}\right)\right| \\
& \leq 4(g(t+\varepsilon)-g(t)) \sum_{j=j_{0}}^{\infty} 3^{-j}=6(g(t+\varepsilon)-g(t)) 3^{-j_{0}}
\end{aligned}
$$

since $\lambda_{x}^{j}, \lambda_{y}^{j} \in(g(t), g(t+\varepsilon))$ for all $j$. Combined with (3.2) this gives $|x-y| \leq$ $c\left|x^{\prime}-y^{\prime}\right|$, where $c$ is as in (3.1). Thus the natural bijection between $C_{g(t)}$ and $A$ is $c$-Lipschitz and we get

$$
\mathcal{H}^{1}(A) \leq c \mathcal{H}^{1}\left(C_{g(t)}\right)=c t<c \mu[0,1] .
$$

By a similar reasoning we see that $\left|x^{\prime \prime}-y^{\prime \prime}\right| \leq c|x-y|$ if $x^{\prime \prime}, y^{\prime \prime} \in C_{g(t+\varepsilon)}$ for which $\operatorname{proj} x=\operatorname{proj} x^{\prime \prime}$ and $\operatorname{proj} y=\operatorname{proj} y^{\prime \prime}$. This gives $c \mathcal{H}^{1}(A) \geq \mathcal{H}^{1}\left(C_{g(t+\varepsilon)}\right)=$ $t+\varepsilon>\mu[0,1]$ and together with (3.3) completes the proof.

We may now finish the proof of Theorem 1.1. Let $1<t_{0}<t_{\max }, A_{t_{0}}=$ $\Theta^{-1}\left(t_{0}, t_{\max }\right)$, and $\delta>0$. Since $g$ is non-decreasing we may cover all, except possibly at most countably many, points of $\left(t_{0}, t_{\max }\right)$ by pairwise disjoint intervals $\left(t, t^{\prime}\right)$ such that $g\left(t^{\prime}\right)-g(t)<\delta$. Lemma 3.1 then implies that $\left.\frac{1}{c} \mu\right|_{A_{t_{0}}} \leq\left.\left(\left.\operatorname{proj} \mathcal{H}^{1}\right|_{A}\right)\right|_{A_{t_{0}}} \leq$ $\left.c \mu\right|_{A_{t_{0}}}$, where $c=1+54 \delta / \min \left\{1,3 g\left(t_{0}\right)\right\}$ (recall that $\Theta^{-1}\{t\}$ has measure zero for all $t$ ). Letting first $\delta \downarrow 0$ and then $t_{0} \downarrow 1$ we get $\mu=\left.\operatorname{proj} \mathcal{H}^{1}\right|_{A}$. This proves the theorem for $\mu$ having a continuous density whose level sets are of measure zero.

For a general $\mu$ there are at most countably many values $t_{n}$ for which $B_{n}=$ $\Theta^{-1}\left\{t_{n}\right\}$ has positive measure and letting $A_{n}=C_{g\left(t_{n}\right)} \cap \operatorname{proj}^{-1} B_{n}$ we have $\left.\mu\right|_{B_{n}}=$ proj $\left.\mathcal{H}^{1}\right|_{A_{n}}$. (If $t_{n}=1$ we cannot use $C_{0}=[0,1] \subset \mathbb{R}^{2}$ since it is rectifiable, but one easily finds a purely 1-unrectifiable set $A_{0} \subset \mathbb{R}^{2}$ for which proj $\left.\mathcal{H}^{1}\right|_{A_{0}}=\mathcal{L}$.) Let $B=[0,1] \backslash \bigcup_{n} B_{n}$. We now use Lusin's Theorem to find a compact set $K_{1} \subset B$ with $\mu\left(B \backslash K_{1}\right)<\frac{1}{2}$ such that $\left.\Theta\right|_{K_{1}}$ is continuous. Then we extend $\left.\mu\right|_{K_{1}}$ to a measure $\nu$ with continuous density whose level sets are of measure zero. The above argument now gives us a purely 1-unrectifiable set $A \subset \mathbb{R}^{2}$ with proj $\left.\mathcal{H}^{1}\right|_{A}=\nu$ and letting $A^{1}=A \cap \operatorname{proj}^{-1}\left(K_{1}\right)$ we have proj $\left.\mathcal{H}^{1}\right|_{A^{1}}=\left.\mu\right|_{K_{1}}$. We continue with the same argument and find a set $K_{2} \subset B \backslash K_{1}$ so that $\Theta$ is continuous on $K_{2}$ and $\mu\left(B \backslash\left(K_{1} \cup K_{2}\right)\right)<\frac{1}{4}$. Then we define a purely 1-unrectifiable set $A^{2}$ such that proj $\left.\mathcal{H}^{1}\right|_{A^{2}}=\left.\mu\right|_{K_{2}}$ and so on. Defining finally $A$ as the union of the sets $A_{n}$ and $A^{n}$ we have proj $\left.\mathcal{H}^{1}\right|_{A}=\mu$.

Remark 3.2. The construction proving Theorem 1.1 in the absolutely continuous case easily generalizes to higher dimensions. Thus, for all absolutely continuous measures $\mu$ on $\mathbb{R}^{n}$ with $\lim _{r \downarrow 0} \mu\left(B(x, r) /(2 r)^{n} \geq 1\right.$ for $\mu$-almost all $x$, there is a purely $n$-unrectifiable Borel set $A \subset \mathbb{R}^{n+1}$ such that $\mu=\left.\operatorname{proj} \mathcal{H}^{n}\right|_{A}$. Here $\operatorname{proj}\left(x_{1}, \ldots, x_{n}, x_{n+1}\right)=\left(x_{1}, \ldots, x_{n}\right)$ and $\mathcal{H}^{n}$ is the non-normalized Hausdorff $n$ measure. We do not have a characterization for the singular case in higher dimensions although we conjecture that a singular measure $\mu$ on $\mathbb{R}^{n}$ may be expressed as $\left.\operatorname{proj} \mathcal{H}^{n}\right|_{A}$ for some purely $n$-unrectifiable $A \subset \mathbb{R}^{n+1}$ if and only if $\mu$ itself is purely $(n-1)$-unrectifiable in the sense that $\mu(B)=0$ for all $(n-1)$-rectifiable sets $B \subset \mathbb{R}^{n}$. 


\section{REFERENCES}

[Fa] K. J. Falconer, The Geometry of Fractal Sets, Cambridge University Press, Cambridge, 1986. MR867284 (88d:28001)

[Ma] P. Mattila, Geometry of Sets and Measures in Euclidean Spaces. Fractals and Rectifiability, Cambridge University Press, Cambridge, 1995. MR1333890(96h:28006)

Department of Mathematics, University College London, Gower Street, London WC1E 6BT, UnITED KINGDOM

E-mail address: mari@math.ucl.ac.uk

Department of Mathematics and Statistics, University of Jyväskylä, P.O. Box 35 (MaD), FIN-40014 Jyvëskylä, Finland

E-mail address: visuomal@maths.jyu.fi 